\title{
Quantifying intervention-related improvements in exercise tolerance
}

\author{
B.J. Whipp and S.A. Ward
}

ABSTRACT: Among the benefits expected to result from a therapeutic intervention in patients with impaired systemic functioning is an increase in exercise tolerance. For this a constant highintensity work rate has been shown to provide a more sensitive index of improvement than the maximum work rate, or oxygen uptake, on a symptom-limited incremental test.

However, the extremely large variability of the improvement in this particular index of tolerance undermines the ability to make general inferences for the underlying functional improvement. We argue that this is a necessary consequence of the particular work rate chosen for the test and the change in the parameters of the subject's hyperbolic power-duration relationship for that form of exercise: its "critical power" and "curvature constant".

Without knowledge of these features, any absolute or per cent increase in tolerance time to a single constant-load exercise bout must be interpreted with caution regarding the physiological benefit(s) that have accrued from the intervention.

KEYWORDS: Critical power, endurance test, power-duration relationship, therapeutic intervention, tolerance time

$\mathbf{T}$ he extent to which a therapeutic strategy improves exercise tolerance in a subject with impaired physiological function is considered to be a reflection of both the utility of the intervention and of its effectiveness. This, however, raises the issues of: 1) which test is employed to assess the improvement; 2) does it matter which; and 3) to what extent does the improvement, as quantified, provide a valid index of improved physiological functioning? We believe it to be important to consider these questions in the light of current recommendations and practice.

\section{CURRENT RECOMMENDATIONS}

In the American Thoracic Society/American College of Chest Physicians statement on cardiopulmonary exercise testing, the authors recognised that "although the incremental protocol is most widely used in clinical practice, constant work rate protocols are gaining popularity because of their clinical applicability, particularly for monitoring response(s) to therapy" [1]. The more recent European Respiratory Society recommendations went further, judging that "in COPD, there is an increasing evidence that high-intensity constant-load endurance protocols ... are superior to other protocols $\left(\right.$ e.g. $V^{\prime} \mathrm{O}_{2}$ peak on maximal intemental test, distance on 6MWT) in the evaluation of the effects of therapeutic interventions" [2].

These recommendations were based on the demonstrations that the improvement in the maximum work rate attained on the ramp-type incremental exercise test, in response to a particular intervention, was relatively small and often close to the limit of the "minimum clinically important difference" [3]. In contrast, the change in time to the limit of tolerance $\left(t_{\mathrm{LIM}}\right)$ on a constant-load test, chosen to be a high fraction of the maximum incremental work rate, was shown to be appreciably larger and hence considered to be more discriminatory, to the extent that investigators now often forego the post-intervention maximum incremental test [4-6]. While the concept is similarly applicable to interventions such as training in normal subjects, it is most commonly utilised, and possibly most consequential, in patients with, for example, chronic obstructive pulmonary disease (COPD). OGA et al. [7] demonstrated in subjects with COPD an average increase of $\sim 20 \%$ in constant-load test duration in response to the inhaled anticholinergic bronchodilator oxytropium bromide, compared with only an $\sim 5 \%$ increase in the

\section{AFFILIATIONS}

Human Bio-Energetics Research Centre, Crickhowell, Powys, UK.

CORRESPONDENCE

B.J. Whipp

Human Bio-Energetics

Research Centre

New Road

Crickhowell

Powys

NP8 1AT

UK

E-mail: bjwhipp@dsl.pipex.com

Received:

July 202008

Accepted after revision:

February 022009

STATEMENT OF INTEREST

None declared. 
maximum incremental work rate. The findings of PORSZASZ et al. [8] are, perhaps, even more striking: endurance training resulted in an average increase in the maximum incremental work rate of $\sim 20 \%$, compared with $\sim 300 \%$ average increase in tolerance to a constant-load test. While others have reported qualitatively similar results [9-13], the actual improvement, in quantitative terms, evidently has an extremely wide range. Naturally, the severity of the impairment and the nature and dose of the intervention play a significant role in this variability. However, the current authors would like to suggest that the choice of the exercise test itself is also a major contributor; sufficiently so that the proportional improvement in the constant-load duration may not be appropriately representative of the actual improvement of physiological functioning resulting from the intervention.

\section{LIMITATIONS OF CURRENT PRACTICE}

The first concern relates to the widely used "percentage of the maximum work rate achieved on the incremental ramp" as the "normalised" intensity for the constant load test. During the non-steady state, the relationship between the ventilatory, skeletal muscle and pulmonary gas exchange responses and the imposed work rate is not constant; it varies as a result of the characteristics of the systems' dynamics [14]. This is schematised for oxygen uptake $\left(V^{\prime} \mathrm{O}_{2}\right)$ in figure 1 , which is based on empirical data such as those from ramp testing by MORTON et al. [15] and for small-step increments (of varying duration) by WHIPP (e.g. [14]).

Note that, as a necessary consequence of the $V^{\prime} \mathrm{O}_{2}$ kinetics, the "lag" in the $V^{\prime} \mathrm{O}_{2}$ response with respect to the steady-state equivalent for that work rate during a ramp-type incremental exercise test (i.e. the vertical distance between the angled solid line and the angled dashed line in figure 1) results in the absolute value of $V^{\prime} \mathrm{O}_{2}$ at any particular work rate becoming progressively less the faster the incrementation rate [14, 16, 17]. Consequently, while the maximally-attained $V^{\prime} \mathrm{O}_{2}$ is not

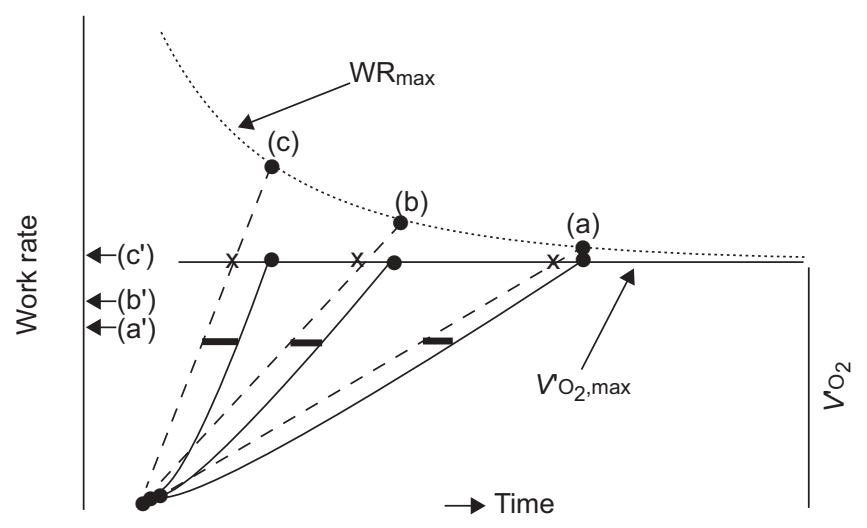

FIGURE 1. Schematic representation of the oxygen uptake $\left(V^{\prime} O_{2}\right)$ response to ramp incremental exercise (-) as a function of time at three different work-rate incrementation rates (- - - ). Note that maximal $V^{\prime} \mathrm{O}_{2}\left(V^{\prime} \mathrm{O}_{2}\right.$, max) is unaffected by workrate incrementation rate. However, the influence of the $V^{\prime} \mathrm{O}_{2}$ time constant (thicker lines) results in the work rate at maximum $\left(\mathrm{WR}_{\max }\right)$ becoming progressively higher, the faster the incrementation rate. The symbols $\left(a^{\prime}\right),\left(b^{\prime}\right)$ and $\left(c^{\prime}\right)$ represent the same percentage of the maximum work rates achieved at the different ramp slopes, i.e. (a), (b) and (c). Modified from [14] with permission from the publisher appreciably (if at all) affected, the maximum work rate attained is (i.e. meeting the criterion for maximum $\left.V^{\prime} \mathrm{O}_{2}[18,19]\right)$. While this has been demonstrated in normal subjects [14,16, 17, 20], a similar body of confirming evidence is not currently available for ramp incremental exercise in patients with COPD, although the results of a range of constant work rate tests [21, 22] suggests that this is likely to be the case. In addition, these subjects may reach the limit of tolerance for reasons different from those of "normal" subjects, in that while "leg fatigue" (and more specifically contractile fatigue, as demonstrated by SAEY et al. [23]) is the major reported cause in a significant number of such patients, dyspnoea is also a predominant contributor (e.g. [23, 24]).

The imposition of a constant work rate at a particular percentage of the maximum value achieved on the incremental ramp test will, therefore, result in a greater metabolic stress and reduced tolerance time for more rapid incrementation rates than for slower ones, as depicted by the arrows $\left(c^{\prime}\right),\left(b^{\prime}\right)$ and $\left(a^{\prime}\right)$ in figure 1. And, as the current optimisation recommendations target a duration of $\sim 10 \mathrm{~min}$ for the incremental phase of the test $[1,2,25,26]$, then different ramp rates are chosen as a function of the known or predicted limit of the subject's aerobic functioning. This "problem" may, to a large extent, be obviated by using the estimated steady-state equivalent work rate of the maximum $V^{\prime} \mathrm{O}_{2}$ rather than the actual work rate attained at maximum $V^{\prime} \mathrm{O}_{2}$. If the subject's "effective" time constant [27] or mean response time [28] for the $V^{\prime} \mathrm{O}_{2}$ kinetics is known (however, it rarely is, and requires prior tests for its determination) or is estimated "broad-brush", then one can simply choose the work rate at that time earlier than the maximum (i.e. the cross in figure 1). A simpler expedient is to draw a line, beginning at the start of the incremental phase of the test, parallel to the linear portion of the ramp $V^{\prime} \mathrm{O}_{2}$ response; where this meets the maximum $V^{\prime} \mathrm{O}_{2}$ value is the required work rate (i.e. the cross in figure 1) [14]. However, as the ramp slopes used in patients with cardiovascular and/or pulmonary disease are typically low $[1,2,25,26]$, the correction for selecting an appropriately normalised constant work rate is relatively small, although still not without consequence for its tolerable duration. But while the maximum $V^{\prime} \mathrm{O}_{2}$-equivalent work rate chosen in this manner provides an improvement over the actual maximum work rate attained on the ramp, it does not completely correct it, as it does not take into account the "slow phase" of the $V^{\prime} \mathrm{O}_{2}$ kinetics manifest at high work rates [29-31], which will shorten the tolerance time on the constant load test. This influence cannot be accurately predicted from the ramp $V^{\prime} \mathrm{O}_{2}$ profile. Also, regardless of the particular percentage of the maximal incremental work rate or $V^{\prime} \mathrm{O}_{2}$ that is chosen for the constant load test, there is likely to be a large intersubject variability in its equivalent intensity domain $[32,33]$. This results in a large intersubject variability in the magnitude and profile of the functional correlates of fatigue, such as: the slow phase of the $V^{\prime} \mathrm{O}_{2}$ kinetics [29-31]; muscle and blood lactate [34, 35] and $\mathrm{pH}$ (e.g. [36]); and the muscle mono-basic phosphate concentration (e.g. [37]).

A further, and even greater, concern regarding the assessment of physiological benefit that can be inferred from the improvement in constant load tolerance time is the relationship 
of the particular chosen work rate to the profile of the subject's power-duration capabilities.

\section{THE POWER-DURATION RELATIONSHIP}

Each subject, if appropriately exercised to the limit of tolerance, has a particular pattern with which the tolerable duration of a constant load exercise bout decreases the greater the work rate; this is characteristic of, and hence characterises, the current state of physiological (predominantly aerobic systems) functioning. The pattern has been widely demonstrated to be that of a rectangular hyperbola [38-41] with an asymptote on the power axis termed the critical power and a curvature constant conventionally termed $\mathrm{W}^{\prime}$, as shown in the following equation, in which WR refers to the work rate and $\mathrm{CP}$ is the critical power.

$$
\mathrm{WR}=\left(\mathrm{W}^{\prime} / t_{\mathrm{LIM}}\right)+\mathrm{CP}
$$

While we focused on cycle ergometry in the present analysis, because much of the clinical testing in this regard has been on the cycle, the concept itself is appropriate for any form of exercise that is performed to the limit of tolerance over a range of work rates. It has, for example, also been supported for running [40], swimming [42], kayaking [43] and, originally, for small-muscle finger flexion exercise [38], and, more recently, for walking in patients with COPD [22]. In fact, CASAS et al. [22] interestingly demonstrated that the physiological responses to an encouraged 6-min walk test provided a close correlate of those at critical power for walking in patients with COPD. This provides a useful contribution towards a wholly-justified method of assessment. However, the tolerance time at a given work rate is also a function of $\mathrm{W}^{\prime}$, which can vary considerably in such patients [21, 22].

The critical power is considered to be an important determinant of exercise tolerance as it has been demonstrated to be the highest constant work rate that can be sustained without $V^{\prime} \mathrm{O}_{2}$, blood and muscle lactate and $\mathrm{H}^{+}$concentrations, and muscle inorganic phosphate, for example, increasing inexorably to or towards maximal attainable levels $[39,44,45]$, i.e. an important parameter of the aerobic energy transfer system. The curvature constant $W^{\prime}$, with the intriguing unit of work, is viewed as being an index of a constant "amount" of energy that is available, or at least that can be utilised, above critical power.

However, if the work rate is plotted as a function of $t_{\mathrm{LIM}}{ }^{-1}$, rather than $t_{\text {LIM }}$ itself, then the linearity of the response supports the hyperbolic nature of the relationship, as shown in figure 2 for
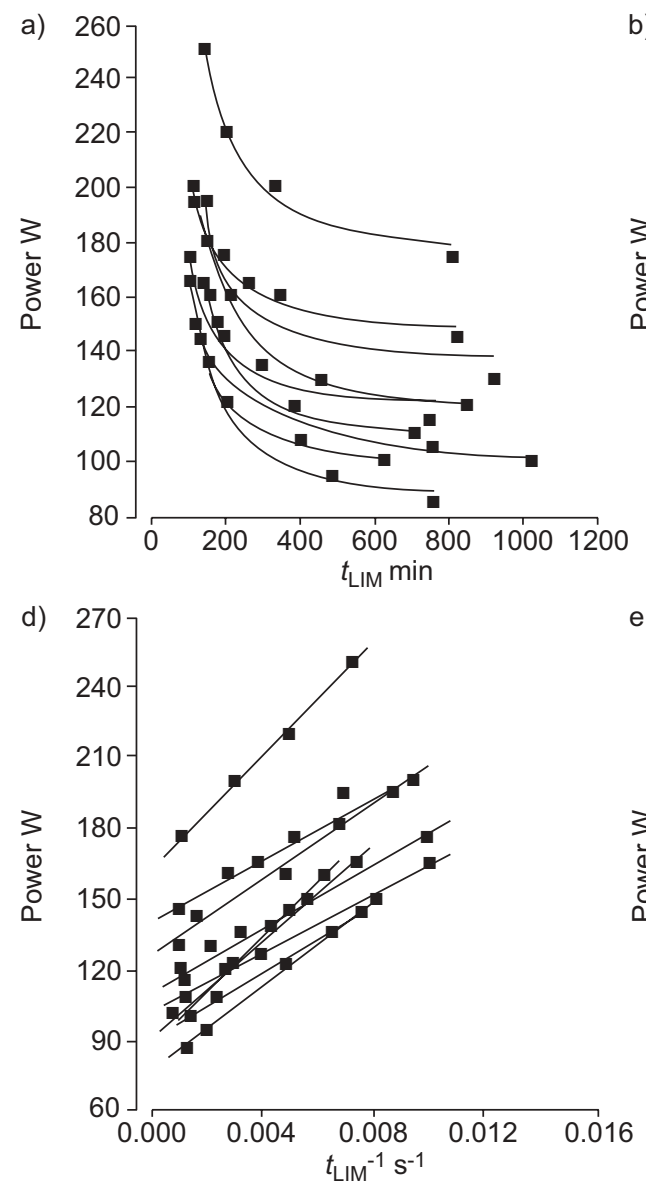
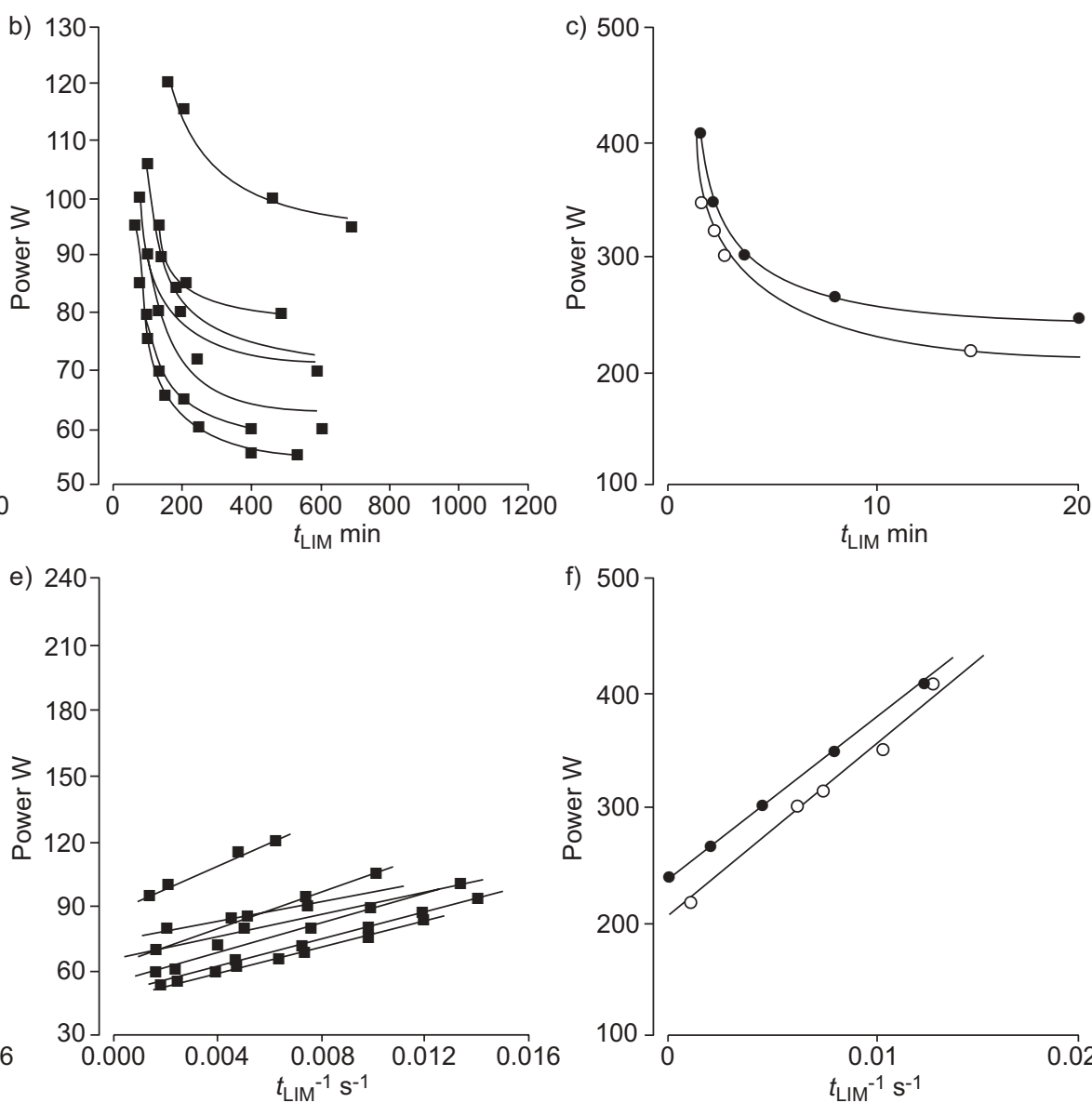

FIGURE 2. The power-duration relationship in a group of a) normal controls and b) subjects with chronic obstructive pulmonary disease (COPD). The power plotted as a function of the reciprocal of the tolerable time for $\mathrm{d}$ ) normal controls and e) subjects with COPD. Modified from [21] with permission from the publisher. $\mathrm{c}$ and $\mathrm{f}$ ) The effect of endurance training on these relationships in a group of normal subjects. $\bigcirc$ : pre-training; $\bullet$ : post-training. $t_{\text {LIM }}$ : time to limit of tolerance. Modified from [9] with permission from the publisher. 
both normal subjects [21, 39] and patients with COPD [21, 46]; critical power being the intercept on the work rate axis and $\mathrm{W}^{\prime}$ being the slope of the relationship. Figure 2 also demonstrates the finding that endurance training in normal subjects results in an increase in critical power with little or no change in $W^{\prime}$ (fig. 2c and f) $[9,47,48]$. This has also been demonstrated in patients with COPD, although, in this case, a small percentage did, in fact, also increase $W^{\prime}$ [46]. In contrast, subjects undergoing sprint training show an increase solely in $\mathrm{W}^{\prime}$ [49]; a link with exercising muscle mass is suggested by the significant correlation between $W^{\prime}$ for cycle ergometry and thigh circumference [50]. And, herewith, the "problem" with interpreting the improvement in constant load tolerance time, resulting from an intervention, and the actual improvement in physiological functioning.

Consider, for example, figure 3, the parameters and the change of parameter values having been taken as characteristic of the COPD subjects studied by NEDER et al. [21] and PUENTE-MAESTU et al. [46]. The pattern of improvement in tolerance time resulting from the intervention is different depending on whether the change is effected by an increase in critical power (fig, 3a) or in $W^{\prime}$ (fig. 3b).
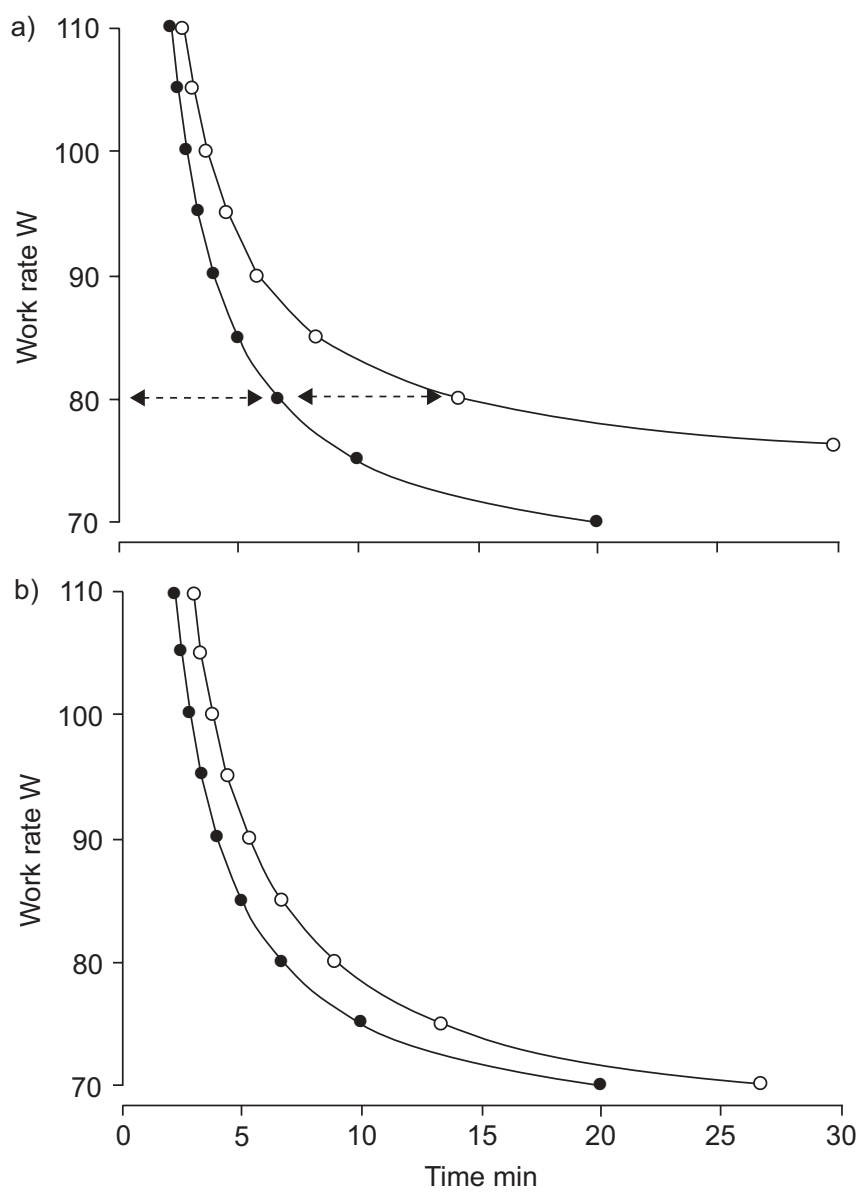

FIGURE 3. a) Schematic representation of the effects of a $10 \%$ increase in critical power on the power-duration relationship. The horizontal arrows reflect the post-intervention work rate for which the tolerance time is doubled (see text for further elaboration). b) The effects of an increase in the curvature constant ( $\left.W^{\prime}\right)$ from 6 to $8 \mathrm{~kJ}$. - : pre-intervention; $\bigcirc$ : post-intervention.
It has been demonstrated that endurance training $[47,48]$ or hypoxia $[51,52]$ modify critical power with little or no change in $\mathrm{W}^{\prime}$, whereas sprint training [49], creatine loading (e.g. [53]) and glycogen depletion [54] result in changes in $W^{\prime}$ with little or no change in critical power. Consequently, although the characteristics of the power-duration relationship are of interest for any cause of limitation of maximal exercise tolerance, the inferences drawn from an improvement following the intervention are likely to be different in different conditions.

While the absolute increase is progressively greater the longer the pre-intervention tolerance time in each case, the increase normalised to the pre-intervention tolerance time, i.e. the conventionally used percentage increase, is quite different, as shown in figure 4.

Changes in $\mathrm{W}^{\prime}$ bring about a constant, and relatively small, increase in the proportional improvement in tolerance. An increase in critical power, however, results in an improvement that is highly dependent on the pre-exercise tolerance time at the particular (and often widely varying) work rate, a point previously made by CASABURI [3]. So much so, in fact, that we contend that it precludes the use of the improvement in tolerance time per se as a valid index of the physiological benefit provided by the intervention. Consider, for example, two subjects (e.g. 1 and 2 in figure 4 ) for whom the intervention has resulted in precisely the same physiological improvement, as reflected by the entire power-duration curve having been shifted upward by a $10 \%$ increase in critical power without, in this case, a change in $\mathrm{W}^{\prime}$. Had the chosen pre-intervention work rate yielded tolerance times of $\sim 5$ and $8 \mathrm{~min}$, for example, then the improvement in $t_{\mathrm{LIM}}$ would be $\sim 70 \%$ in one case and $\sim 200 \%$ in the other. But in the latter case, this could be appreciably greater with an even longer preintervention tolerance time, as the per cent improvement curve (fig. 4) asymptotes to infinity. But an even more disquieting outcome is represented by considering subjects 2 and 3 . In this case, with a $10 \%$ increase in critical power,

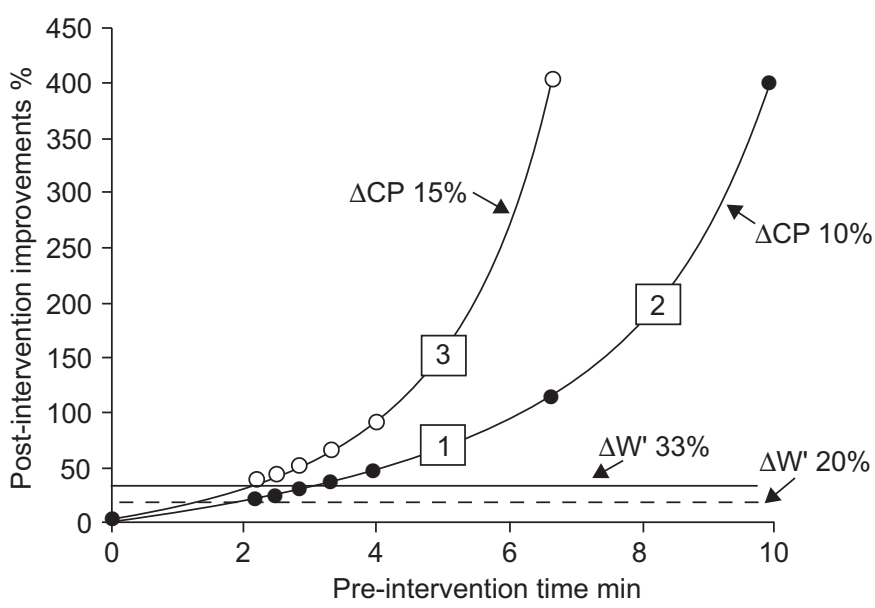

FIGURE 4. Schematic representation of the effects of a $10 \%$ and $15 \%$ increase in critical power (CP) and a $20 \%$ and $33 \%$ increase in the curvature constant $\left(\mathrm{W}^{\prime}\right)$ on an intervention-induced percentage increase in the tolerable duration to a series of constant work-rate tests as a function of the pre-intervention tolerance time. 1,2 and 3 depict three particular percentage improvements for comparison (see text for further elaboration). 
subject 2 has had a "poorer" physiological response to the intervention than subject 3, who has benefited from a $15 \%$ increase. But, when considered in terms of the improvement in $t_{\text {LIM }}$, subject 2 would actually "seem" to have had a "better" response to the intervention; not so, of course.

\section{TOWARDS AN IMPROVED INTERPRETATION}

Although the test-retest reproducibility of critical power has been shown to be high in normal subjects [47, 55], with correlation coefficients $>0.9$, no such evidence is currently available in patients with COPD. What is available, however, are a few small-scale cycle ergometer studies on reproducibility of a single high-intensity work rate; these have concluded that reproducibility is high [56-58]. More work is needed, however, on the influence of individual point deviation on the parameter estimates of the power-duration curve and its implications for interpretation.

While recognising that an improvement in exercise tolerance to any particular constant work rate is indeed reflective of a physiological benefit of the intervention, it is not of itself adequate as an appropriate quantitative index of the actual improvement in function, as for a change in the value of a variable of interest at a chosen "iso-time" $[4,5,59]$ that provides a single point on a usually increasing profile of response. Ideally, the pre- and post-intervention powerduration characteristics would be necessary. At present, however, this cannot be established with an "appropriately simple" test. And even were this to have been established, then what quantitative index of the improvement would be chosen to be both practical and justifiable? One approach, having

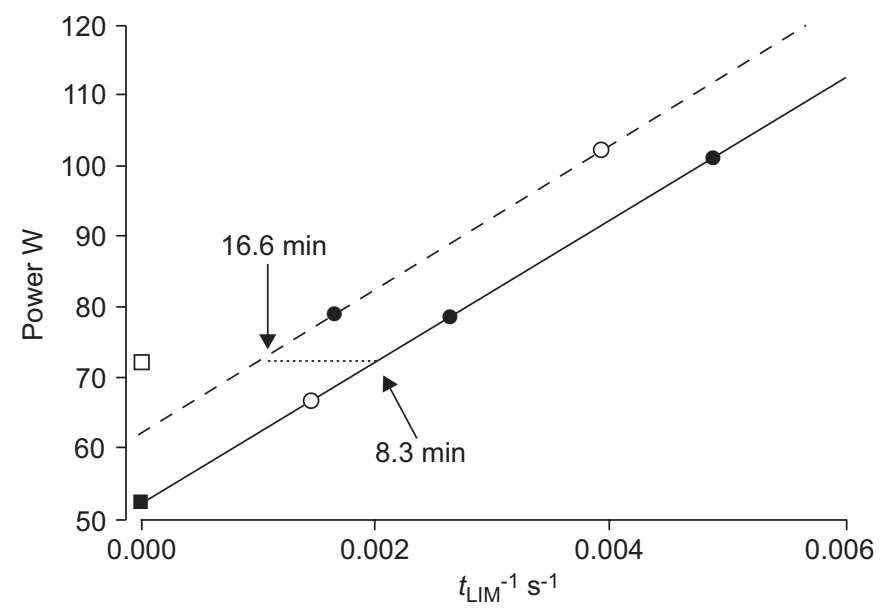

FIGURE 5. Schematic representation of the power-inverse tolerance time relationship for high intensity, constant work rate exercise. The solid symbols represent the minimum number of tests to estimate the subject's pre-intervention critical power ( $\mathbf{\square})$ and curvature constant for the power-duration relationship ( - ); $\bigcirc$ represents an additional "confirmation" test, if needed. The post-intervention relationship (- - - - ) is constructed as a line through a single experimentally determined point $(\bullet)$ and parallel to the pre-intervention line; $\bigcirc$ represents an additional point to be established if the "parallel assumption" is judged to be inadequate for the purpose. $\cdots \cdot$ : the work rate at which there is a doubling of the tolerance time $(\square)$ as a result of the intervention, resulting, in this case, in a $38 \%$ improvement: per cent change in work rate when tolerance time is doubled is ((73$53) / 53) \times 100=38 \%$. t LIM: time to limit of tolerance. similarities to the concepts of rheobase and chronaxie in neurophysiology $[60,61]$, might be promising, where rheobase is the minimum applied current strength (i.e. the asymptotic stimulus) that will produce a response (i.e. nerve "firing") and chronaxie is the stimulus duration that elicits a response when the stimulus strength is twice that of rheobase. But, of course, determining the exercise tolerance time at a work rate which is double that of the critical power is quite inappropriate for clinical exercise testing purposes: it is simply too high. There are also concerns with using lower percentages, prominent among which is the possibility that the critical power might increase by an even greater percentage. And so, as a practical expedient, one might consider the post-intervention work rate that results in a doubling of the pre-intervention tolerance time at that work rate (fig. 3), and normalised as a percentage of the pre-intervention critical power. This has the advantage of being able to be established for all subjects, regardless of the magnitude of the intervention-induced increase in critical power itself.

But, can this be done "simply"? Possibly not; at least not simply enough for the usual demands of "clinical exercise testing" $[62,63]$. We suggest one approach (others, naturally, may evolve) that may provide an improvement in the current methodology. This would involve two pre-intervention $t_{\text {LIM }}$ tests being performed, as outlined in figure 5 (the solid symbols on the solid line) [64]. From this, and assuming that each test was a valid maximum effort, both critical power and $\mathrm{W}^{\prime}$ can be determined, with the recognition that the confidence of the parameter estimation is increased the greater the number of valid $t_{\text {LIM }}$ values used (e.g. adding the open circle value in figure 5). Post-intervention, the minimum number is one. If, that is, the investigator is prepared to assume that $\mathrm{W}^{\prime}$ has not changed, as has been demonstrated for endurance training in normal subjects and, in most cases, in patients with COPD. That is, a line (dashed line in figure 5) through the determined $t_{\text {LIM }}$ point (closed circle) parallel to the preintervention slope (i.e. unchanged $W^{\prime}$ ) extrapolates to the post-intervention critical power. If that assumption is judged to be inappropriate then, of necessity, a further $t_{\text {LIM }}$ point (e.g. in figure 5 the open circle) is a necessary minimum to estimate the parameters. The post-intervention work rate that results in a doubling of the pre-intervention tolerance time can then be determined (as shown by the horizontal dotted line in figure 5) and used as an index of the functional improvement wrought by the intervention.

While this complicates the testing somewhat, it is necessary to recognise that without this, or a similar conceptually justifiable index, any absolute or per cent increase in tolerance time to a single constant load exercise bout must be interpreted with caution regarding the physiological benefit(s) that have accrued from the intervention.

\section{REFERENCES}

1 American Thoracic Society, American College of Chest Physicians. ATS/ACCP statement on cardiopulmonary exercise testing. Am J Respir Crit Care Med 2003; 167: 211-277.

2 Palange P, Ward SA, Carlsen K-H, et al. Recommendations on the use of exercise testing in clinical practice. Eur Resp J 2007; 29: 185-209. 
3 Casaburi R. Factors determining constant work rate exercise tolerance in COPD and their role in dictating the minimal clinically important difference in response to interventions. COPD 2005; 2: 131-136.

4 O'Donnell DE, Voduc N, Fitzpatrick M, et al. Effect of salmeterol on the ventilatory response to exercise in chronic obstructive pulmonary disease. Eur Respir J 2004; 24: 86-94.

5 Palange P, Valli G, Onorati P, et al. Effect of heliox on lung dynamic hyperinflation, dyspnea, and exercise endurance capacity in COPD patients. J Appl Physiol 2004; 97: 16371642.

6 van't Hul A, Gosselink R, Hollander P, et al. Training with inspiratory pressure support in patients with severe COPD. Eur Respir J 2006; 27: 65-72.

7 Oga T, Nishimura K, Tsukino M, et al. The effects of oxitropium bromide on exercise performance in patients with stable chronic obstructive pulmonary disease. A comparison of three different exercise tests. Am J Respir Crit Care Med 2000; 161: 1897-1901.

8 Porszasz J, Emtner M, Goto S, et al. Exercise training decreases ventilatory requirements and exercise-induced hyperinflation at submaximal intensities in patients with COPD. Chest 2005; 128: 2025-2034.

9 Poole DP, Ward SA, Whipp BJ. Effect of training on the metabolic and respiratory profile of heavy and severe exercise. Eur J Appl Physiol 1990; 59: 421-429.

10 Ortega F, Toral J, Cejudo P, et al. Comparison of effects of strength and endurance training in patients with chronic obstructive pulmonary disease. Am J Respir Crit Care Med 2002; 166: 669-674.

11 Neder JA, Sword D, Ward SA, et al. Home based neuromuscular electrical stimulation as a new rehabilitative strategy for severely disabled patients with chronic obstructive pulmonary disease (COPD). Thorax 2002; 57: 333-337.

12 Costes F, Agresti A, Court-Fortune I, et al. Noninvasive ventilation during exercise training improves exercise tolerance in patients with chronic obstructive pulmonary disease. J Cardiopulm Rehabil 2003; 23: 307-313.

13 Emtner M, Porszasz J, Burns M, et al. Benefits of supplemental oxygen in exercise training in nonhypoxemic chronic obstructive pulmonary disease patients. Am J Respir Crit Care Med 2003; 168: 1034-1042.

14 Whipp BJ. The bioenergetic and gas-exchange basis of exercise testing. Clin Chest Med 1994; 15: 173-192.

15 Morton RH, Green S, Bishop D, et al. Ramp and constant power trials produce equivalent critical power estimates. Med Sci Sports Exerc 1997; 29: 833-836.

16 Davis JA, Whipp BJ, Huntsman DJ, et al. Criteria for establishing the slope of ramp work rate exercise tests. Med Sci Sports 1982; 14: 339-343.

17 Hansen JE, Sue DY, Oren A, et al. Relation of oxygen uptake to work rate in normal men and men with circulatory disorders. Am J Cardiol 1987; 59: 669-674.

18 Taylor HL, Buskirk E, Henschel A. Maximal oxygen intake as an objective measure of cardiorespiratory performance. J Appl Physiol 1955; 8: 73-80.

19 Mitchell JH, Sproule BJ, Chapman CB. The physiological meaning of the maximal oxygen intake test. J Clin Invest 1957; 37: 538-547.
20 Scheuermann BW, Kowalchuk JM. Attenuated respiratory compensation during rapidly incremented ramp exercise. Respir Physiol 1998; 114: 227-238.

21 Neder JA, Jones PW, Nery LE, et al. Determinants of the exercise endurance capacity in patients with chronic obstructive pulmonary disease. The power-duration relationship. Am J Respir Crit Care Med 2000; 162: 497-504.

22 Casas A, Vilaro J, Rabinovich R, et al. Encouraged 6-min walking test indicates maximum sustainable exercise in COPD patients. Chest 2005; 128: 55-61.

23 Saey D, Debigare R, LeBlanc P, et al. Contractile leg fatigue after cycle exercise: a factor limiting exercise in patients with chronic obstructive pulmonary disease. Am J Respir Crit Care Med 2003; 168: 425-430.

24 Killian KJ, LeBlanc P, Martin DH, et al. Exercise capacity and ventilatory, circulatory, and symptom limitation in patients with airflow limitation. Am Rev Respir Dis 1992; 146: 935-940.

25 Wasserman K, Hansen JE, Sue DY, et al. Principles of Exercise Testing and Interpretation. 4th Edn. Philadelphia, Lea \& Febiger, 2005.

26 Cooper CB, Storer TW. Exercise Testing and Interpretation. Cambridge, Cambridge University Press, 2001.

27 Whipp BJ, Ward SA. Physiological determinants of pulmonary gas exchange kinetics during exercise. Med Sci Sports Ex 1990; 22: 62-71.

28 Linnarsson D. Dynamics of pulmonary gas exchange and heart rate changes at start and end of exercise. Acta Physiol Scand 1974; 415: Suppl., 1-68.

29 Whipp BJ, Ward SA, Rossiter HB. Pulmonary $\mathrm{O}_{2}$ uptake during exercise: conflating muscular and cardiovascular responses. Med Sci Sports Exerc 2005; 37: 1574-1585.

30 Barstow TJ. Characterization of $\mathrm{V}^{\prime} \mathrm{O}_{2}$ kinetics during heavy exercise. Med Sci Sports Exerc 1994; 26: 1327-1334.

31 Jones AM, Poole DC. Oxygen uptake dynamics: from muscle to mouth - an introduction to the symposium. Med Sci Sports Exerc 2005; 37: 1542-1550.

32 Whipp BJ, Rossiter HB, Ward SA. Exertional oxygen uptake kinetics: a stamen of stamina? Biochem Trans 2002; 30: 237-247.

33 Hansen JE, Sue DY, Wasserman K. Predicted values for clinical exercise testing. Am Rev Respir Dis 1984; 129: Suppl, S49-S55.

34 Hogan MC, Gladden LB, Kurdak SS, et al. Increased [lactate] in working dog muscle reduces tension development independent of pH. Med Sci Sports Exerc 1995; 27: 371-377.

35 Favero TG, Zable AC, Colter D, et al. Lactate inhibits $\mathrm{Ca}^{2+}$ activated $\mathrm{Ca}^{2+}$-channel activity from skeletal muscle sarcoplasmic reticulum. J Appl Physiol 1997; 82: 447-452.

36 Fitts RH. Cellular mechanisms of muscle fatigue. Physiol Rev 1994; 74: 49-94.

37 Westerblad H, Allen DG, Lännergren J. Muscle fatigue: lactic acid or inorganic phosphate the major cause? News Physiol Sci 2002; 17: 17-21.

38 Monod H, Scherrer J. The work capacity of a synergic muscular group. Ergonomics 1965; 8: 329-338.

39 Poole DC, Ward SA, Gardner GW, et al. Metabolic and respiratory profile of the upper limit for prolonged exercise in man. Ergonomics 1988; 31: 1265-1279. 
40 Hughson RL, Orok CK, Staudt LE. A high velocity treadmill running test to assess endurance running potential. Int J Sports Med 1984; 5: 23-25.

41 Hill DW. The critical power concept: a review. Sports Med 1993; 16: 237-254.

42 Wakayoshi K, Ikuta K, Yoshida T, et al. Determination and validity of critical velocity as an index of swimming performance in the competitive athlete. Europ J Appl Physiol 1992; 64: 153-157.

43 Nakamura FY, Borges TO, Sales OR, et al. Energetic cost estimation and contribution of different metabolic pathways in speed kayaking. Rev Bras Med Esporte 2004; 10: 78-84.

44 Whipp BJ. Domains of aerobic function and their limiting parameters. In: Steinacker JM, Ward SA, eds. The Physiology and Pathophysiology of Exercise Tolerance. New York, Plenum, 1996; pp. 83-89.

45 Jones AM, Wilkerson DP, DiMenna F, et al. Muscle metabolic responses to exercise above and below the "critical power" assessed using ${ }^{31} \mathrm{P}$-MRS. Am J Physiol Regul Integr Comp Physiol 2008; 294: R585-R593.

46 Puente-Maestu L, Santa Cruz A, Vargas T, et al. Effects of training on the tolerance to high-intensity exercise in patients with severe COPD. Respiration 2003; 70: 367-370.

47 Gaesser GA, Wilson LA. Effects of continuous and interval training on the parameters of the power-endurance time relationship for high-intensity exercise. Int J Sports Med 1988; 9: 417-421.

48 Poole DP, Ward SA, Whipp BJ. Effect of training on the metabolic and respiratory profile of heavy and severe exercise. Eur J Appl Physiol 1990; 59: 421-429.

49 Jenkins DG, Quigley BM. The influence of high-intensity exercise training on the Wlim-Tlim relationship. Med Sci Sports Exerc 1993; 25: 275-282.

50 Miura A, Endo M, Sato H, et al. Relationship between the curvature constant parameter of the power-duration curve and muscle cross-sectional area of the thigh for cycle ergometry in humans. Eur J Appl Physiol 2002; 87: 238-244.

51 Moritani T, Nagata A, de Vries HA, et al. Critical power as a measure of physical work capacity and anaerobic threshold. Ergonomics 1981; 24: 339-350.

52 Whipp BJ, Huntsman DJ, Storer TW, et al. A constant which determines the duration of tolerance to highintensity work. Fed Proc 1982; 41: 1591.
53 Miura A, Kino F, Kajitani S, et al. The effect of oral creatine supplementation on the curvature constant parameter of the power-duration curve for cycle ergometry in humans. Jpn J Physiol 1999; 49: 169-174.

54 Miura A, Sato H, Sato $\mathrm{H}$, et al. The effect of glycogen depletion on the curvature constant parameter of the power-duration curve for cycle ergometry. Ergonomics 2000; 43: 133-141.

55 Nebelsick-Gullett LJ, Housh TJ, Johnson GO, et al. A comparison between methods of measuring anaerobic work capacity. Ergonomics 1988; 31: 1413-1419.

56 Noseda A, Carpiaux JP, Prigogine T, et al. Lung function, maximum and submaximum exercise testing in COPD patients: reproducibility over a long interval. Lung 1989; 167: 247-257.

57 O'Donnell DE, Lam M, Webb KA. Measurement of symptoms, lung hyperinflation, and endurance during exercise in chronic obstructive pulmonary disease. Am J Respir Crit Care Med 1998; 158: 1557-1565.

58 van't Hul A, Gosselink R, Kwakkel G. Constant-load cycle endurance performance: test-retest reliability and validity in patients with COPD. J Cardiopulm Rehabil 2003; 23: 143-150.

59 Casaburi R, Kukafka D, Cooper CB, et al. Improvement in exercise tolerance with the combination of tiotropium and pulmonary rehabilitation in patients with COPD. Chest 2005; 127: 809-817.

60 Lapicque L. Définition expérimentale de l'excitabilité [Experimental definition of exitability]. Soc Biol 1909; 77: 280-283.

61 Holsheimer J, Dijkstra EA, Demeulemeester $\mathrm{H}$, et al. Chronaxie calculated from current-duration and voltageduration data. J Neurosci Methods 2000; 97: 45-50.

62 Brickley G, Dekerle J, Hammond AJ, et al. Assessment of maximal aerobic power and critical power in a single 90-s isokinetic all-out cycling test. Int J Sports Med 2007; 28: 414-419.

63 Vanhatalo A, Doust JH, Burnley M. Determination of critical power using a 3-min all-out cycling test. Med Sci Sports Exerc 2007; 39: 548-555.

64 Malaguti C, Nery LE, Dal Corso S, et al. Alternative strategies for exercise critical power estimation in patients with COPD. Eur J Appl Physiol 2006; 96: 59-65. 